\title{
Response of the rare biosphere to environmental stressors in a highly diverse ecosystem (Zodletone spring, OK, USA)
}

Suzanne Coveley, Mostafa S Elshahed, Noha H. Youssef

Within highly diverse ecosystems, the majority of bacterial taxa are present in low abundance as members of the rare biosphere. The rationale for the occurrence and maintenance of the rare biosphere, and the putative ecological role(s) and dynamics of its members within a specific ecosystem is currently debated. We hypothesized that in highly diverse ecosystems, a fraction of the rare biosphere acts as a backup system that readily responds to environmental disturbances. We tested this hypothesis by subjecting sediments from Zodletone spring, a sulfide- and sulfur-rich spring in southwestern OK, to incremental levels of salinity $(1,2,3,4$, and $10 \% \mathrm{NaCl})$, or temperature $\left(28^{\circ}, 30^{\circ}, 32^{\circ}\right.$, and $70^{\circ} \mathrm{C}$ ), and traced the trajectories of rare members of the community in response to these manipulations using 16S rRNA gene analysis. Our results indicate that multiple rare bacterial taxa are promoted from rare to abundant members of the community following such manipulations and that, in general, the magnitude of such recruitment is directly proportional to the severity of the applied manipulation. Rare members that are phylogenetically distinct from abundant taxa in the original sample (unique rare biosphere) played a more important role in the microbial community response to environmental disturbances, compared to rare members that are phylogenetically similar to abundant taxa in the original sample (non-unique rare biosphere). The results emphasize the dynamic nature of the rare biosphere, and highlight its complexity and non-monolithic nature. 


\title{
Response of the rare biosphere to environmental stressors in a highly diverse ecosystem (Zodletone spring, OK, USA)
}

\begin{abstract}
Within highly diverse ecosystems, the majority of bacterial taxa are present in low abundance as members of the rare biosphere. The rationale for the occurrence and maintenance of the rare biosphere, and the putative ecological role(s) and dynamics of its members within a specific ecosystem is currently debated. We hypothesized that in highly diverse ecosystems, a fraction of the rare biosphere acts as a backup system that readily responds to environmental stressors. We tested this hypothesis by subjecting sediments from Zodletone spring, a sulfide- and sulfur-rich spring in southwestern $\mathrm{OK}$, to incremental levels of salinity $(1,2,3,4$, and $10 \% \mathrm{NaCl})$, or temperature $\left(28^{\circ}, 30^{\circ}, 32^{\circ}\right.$, and $\left.70^{\circ} \mathrm{C}\right)$, and traced the trajectories of rare members of the community in response to these manipulations using $16 \mathrm{~S}$ rRNA gene analysis. Our results indicate that multiple rare bacterial taxa are promoted from rare to abundant members of the community following such manipulations, and that, in general, the magnitude of such recruitment is directly proportional to the severity of the applied manipulation. Rare members that are phylogenetically distinct from abundant taxa in the original sample (unique rare biosphere) played a more important role in the microbial community response to environmental stressors, compared to rare members that are phylogenetically similar to abundant taxa in the original sample (non-unique rare biosphere). The results emphasize the dynamic nature of the rare biosphere, and highlight its complex nature.

Authors: Suzanne Coveley ${ }^{1}$, Mostafa S. Elshahed ${ }^{1}$, and Noha H. Youssef ${ }^{1 *}$

Affiliations: ${ }^{1}$ Department of Microbiology and Molecular Genetics, Oklahoma State University Stillwater, OK, USA

*Corresponding author. Address: 1110 S. Innovation way Dr., Stillwater, Oklahoma 74074 USA. Phone: 1-405-744-1192, Fax. 1-405-744-1112, email: noha@okstate.edu
\end{abstract}




\section{Introduction}

Microbial communities in nature are extremely diverse. Recent high throughput $16 \mathrm{~S}$

rRNA gene-based sequencing surveys have revealed that microbial communities in highly diverse samples exhibit a distribution pattern where the majority of bacterial species are present in extremely low numbers (Ashby et al. 2007; Bowen et al. 2012; Logares et al. 2014; PedrosAlio 2006; Sogin et al. 2006). This fraction of the microbial community has been referred to as the rare biosphere (Sogin et al. 2006).

Multiple studies have examined various characteristics of the rare biosphere, e.g. its proportional size within a specific sample, phylogenetic affiliations of its members, biogeography and ecology of its members, as well as its spatial and temporal dynamics in a specific habitat (Alonso-Saez et al. 2015; Alonso-Saez et al. 2014; Anderson et al. 2015; Gobet et al. 2012; Hugoni et al. 2013; Liu et al. 2015; Reveillaud et al. 2014; Shade \& Gilbert 2015; Shade et al. 2014; Youssef et al. 2010). All studies invariably suggest that members of the rare biosphere are phylogenetically diverse and could, collectively, mediate multiple metabolic capabilities and ecosystem functions. For example, studies have shown that members of the rare biosphere exhibit a wide range of phylogenetic novelty (Elshahed et al. 2008; Lynch et al. 2012; Pester et al. 2012; Youssef et al. 2012): While a fraction of the rare biosphere is typically novel (at the phylum, class, order, or family levels), many others are closely related to previously described lineages in other ecosystems. Similarly, members of the rare biosphere exhibit various levels of uniqueness (Elshahed et al. 2008; Galand et al. 2009), with a fraction being unique (i.e. bears no resemblance to other members in the community), while others are very closely related to more abundant members of the community. On a functional level, several studies have documented a large variation in the level of metabolic activity of members of the rare biosphere 
(by studying $\mathrm{rRNA} / \mathrm{rDNA}$ transcript to gene ratios) within a single sample (Campbell et al. 2011), ranging from apparent dormancy to disproportionately high metabolic activity (Besemer et al. 2012; Jones \& Lennon 2010; Logares et al. 2014; Pester et al. 2010; Pester et al. 2012; Wilhelm et al. 2014).

Maintenance of members of the rare biosphere within a specific ecosystem suggests that they perform essential ecological functions. As described above, the involvement of members of the rare biosphere in specific metabolic processes within an ecosystem has been documented. Another plausible contribution of the rare biosphere to ecosystem functions is by acting as a backup system that responds to various environmental stressors e.g. temperature and $\mathrm{pH}$ changes, desertification, drastic change in redox potential, hydrocarbon spill (Crump et al. 2012; Elshahed et al. 2008; Lennon \& Jones 2011; Marchant et al. 2002; Taylor et al. 2013; Walke et al. 2014). Under this scenario, environmental stressors could induce the growth and promotion of specific members of the rare biosphere that are metabolically and physiologically more adapted to the new prevailing condition, and this promotion is coupled to the demotion of formerly abundant members in the community inadequately adapted to the new conditions (Elshahed et al. 2008). Such dynamic process contributes to the functional flexibility of a specific ecosystem. It could also explain the observed higher diversity, and functional redundancy in diverse environments, and the retention of the rare biosphere in such systems (Shade et al. 2012; Yachi \& Loreau 1999).

We reason that such hypothesis could be experimentally evaluated by stepwise subjection of a microbial community to a gradient of environmental stressors of varying magnitudes, and observing the associated patterns of promotion and demotion within rare and abundant members of the community. Here, sediments obtained from Zodletone spring, a highly diverse anoxic 
77 spring in southwestern Oklahoma, USA, were subjected to various incremental degrees of

78 salinity or temperature shifts. The microbial community was examined pre and post enrichment

79 to characterize the dynamics of microbial community shifts associated with such processes. Our

80 results support the proposed functionality and role of the rare biosphere in responding to

81 environmental stressors.

82 
Sampling. Sediment samples were collected from Zodletone spring, a sulfide and sulfur-rich spring in southwestern Oklahoma $\left(35^{\circ} 0^{\prime} 9^{\prime \prime} \mathrm{N}, 98^{\circ} 41^{\prime} 17^{\prime \prime} \mathrm{W}\right)$. The spring geochemistry has been previously described in detail (Bühring et al. 2011; Senko et al. 2004). The source of the spring is a contained area $\left(1 \mathrm{~m}^{2}\right)$ with anaerobic, biomass-laden, and sulfide-rich black viscous sediments, and anoxic, sulfide-rich $(8.4 \mathrm{mM}) 40-\mathrm{cm}$ water column. Spring source water was also sampled for use in enrichments as detailed below. Samples were stored on ice until returning to the lab, where they were used for the experimental procedures described below within $24 \mathrm{~h}$ of sampling.

Enrichments preparation. The overall experimental setup is shown in Figure 1.

Enrichments were prepared in Balch tubes under anaerobic conditions in an anaerobic chamber (Coy Laboratories, Grass Lake, MI, USA) by adding $1 \mathrm{~g}$ sediment to $4 \mathrm{ml}$ autoclaved anoxic spring water. For this purpose, spring source water was autoclaved, cooled under a stream of $\mathrm{N}_{2}$ to maintain anaerobic conditions, and used in all enrichments. To mimic environmental stress, either salinity or temperature was varied in the enrichments. Salinity was adjusted by adding $\mathrm{NaCl}$ to increase the concentration to $1 \%, 2 \%, 3 \%, 4 \%$, or $10 \%$ above ambient concentration (measured at $0.9 \%$ ) and these enrichments were incubated at room temperature. Temperature was varied by incubating enrichments at $28^{\circ} \mathrm{C}, 30^{\circ} \mathrm{C}, 32^{\circ} \mathrm{C}$, or $70^{\circ} \mathrm{C}$. For each condition, triplicate enrichments were incubated for 60 days in the dark. To account for any changes that could occur due to the incubation process itself, triplicate control enrichments were prepared by incubating $1 \mathrm{~g}$ sediment in $4 \mathrm{ml}$ autoclaved anoxic spring water with no further changes in salinity or temperature. This no-stressor enrichment control was subsequently used as the baseline for studying the effect of temperature and salinity change on the microbial community. 
DNA extraction, PCR amplification and sequencing. Triplicates were pooled, centrifuged at $10,000 \mathrm{xg}$ for 20 minutes and DNA was extracted from the obtained pellet using MoBio FastDNA Spin kit for Soil (MoBio, Carlsbad, CA) following the manufacturer's instructions, and subsequently quantified using Qubit ${ }^{\circledR}$ fluorometer (Life Technologies, Grand Island, NY). Variable regions V1 and V2 of the 16S rRNA gene were then amplified using barcoded primers for multiplex sequencing using the FLX technology. The forward primer was constructed by adding 454 Roche FLX adaptor A (GCCTCCCTCGCGCCATCAG) to the 27F primer sequence (AGAGTTTGATCCTGGCTCAG) as previously described (Youssef et al. 2010). The forward primer also contained a unique barcode (octamer) sequence for multiplexing. The reverse primer was constructed by adding 454 Roche FLX adaptor B (GCCTTGCCAGCCCGCTCAGT) to the 338R primer sequence (GCTGCCTCCCGTAGGAGT). PCRs were conducted in $100 \mu 1$ volume. The reaction contained $4 \mu 1$ of the extracted DNA, $1 \times$ PCR buffer (Promega, Madison, WI), 2.5 $\mathrm{mM} \mathrm{MgSO}$, $0.2 \mathrm{mM}$ dNTPs mixture, $0.5 \mathrm{U}$ of the GoTaq flexi DNA polymerase (Promega, Madison, WI), and $10 \mu \mathrm{M}$ each of the forward and the reverse primers. PCR was carried out according to the following protocol; initial denaturation at $95^{\circ} \mathrm{C}$ for 5 minutes, followed by 35 cycles of denaturation at $95^{\circ} \mathrm{C}$ for $45 \mathrm{sec}$, annealing at $52^{\circ} \mathrm{C}$ for $45 \mathrm{sec}$, and elongation at $72^{\circ} \mathrm{C}$ for $30 \mathrm{sec}$. A final elongation step at $72^{\circ} \mathrm{C}$ for 5 minutes was included. All PCR reactions were run in at least triplicates, and the resulting products of the expected size were combined and purified using QIAquick PCR cleanup kit (Qiagen Corp., Valencia, CA). Purified combined PCR products (11-15 $\mu \mathrm{g}$, equivalent to $\sim 1 \mu \mathrm{g}$ of each enrichment condition) were sequenced using FLX technology at the Environmental Genomics Core facility at the University of South Carolina. 
Sequence quality filtering, OTU identification, and phylogenetic assignments. Sequence quality control was handled in mothur (Schloss et al. 2009) as described previously (Youssef et al. 2010). Briefly, sequences with an average quality score below 25 , sequences that did not have the exact primer sequence, sequences that contained an ambiguous base $(\mathrm{N})$, sequences having a homopolymer stretch longer than 8 bases, and sequences shorter than $80 \mathrm{bp}$ were considered of poor quality and removed from the data set. High-quality reads from each treatment condition were aligned against the Greengenes alignment database using a Needleman-Wunsch pairwise alignment algorithm. Filtered alignments were used to generate an uncorrected pairwise distance matrix, followed by binning the sequences into operational taxonomic units (OTUs) at 3, 6, 8, 10 , and $15 \%$ cutoffs. For phylogenetic placement, representative OTUs defined at the $3 \%$ cutoff $\left(\mathrm{OTU}_{0.03}\right)$ were classified with Greengenes taxonomy scheme using the PyNAST pipeline (Caporaso et al. 2010). Phylum level affiliation of sequences were determined according to the classifier output, and sequences with less than $85 \%$ similarity to their closest relative in Greengenes database were considered unclassified.

Defining the rare biosphere. The cutoff for defining rare biosphere is arbitrary and the methods used include relative abundance cutoffs, as well as frequencies of occurrence in a dataset (Youssef et al. 2010). For the sake of this study, we used a more relaxed definition for the rare biosphere, where rare members of the microbial community were identified using an empirical cutoff of $n \leq 10$.

\section{Examining microbial community response to environmental stressors.}

Our analysis had two main goals: To identify differences in the overall microbial community structure and diversity pre and post-enrichment, and to examine and quantify the contribution of various members of the rare biosphere in response to environmental stressors. 
For the first goal (i.e. identification of differences in the overall microbial community structure and diversity due to environmental stress), microbial diversity was quantified using various diversity indices e.g. Shannon-Weiner, and Simpson diversity indices, Chao, and ACE estimators of species richness across enrichments. As well, beta diversity across samples was examined using rarefaction curve ranking since this method is not sensitive to sample size as described before (Youssef \& Elshahed 2009). Finally, variations in community structure due to stressors were calculated using Morisita-Horn index. This index was chosen due to its insensitivity to sample size variations (Anderson \& Millar 2004). Morisita-Horn index values obtained were subsequently employed to construct non-metric multidimensional scaling (NMDS) plots for community comparisons using the command nmds in mothur. The proportion of variance $\left(\mathrm{r}^{2}\right)$ among communities was estimated from the NMDS plots by first calculating the Euclidean distance between all pairs of data points using the equation;

$d=\sqrt{\left(x_{2}-x_{1}\right)^{2}+\left(y_{2}-y_{1}\right)^{2}}$, where $\mathrm{d}$ is the Euclidean distance between 2 points of coordinates $\left(\mathrm{x}_{1}, \mathrm{y}_{1}\right)$ and $\left(\mathrm{x}_{2}, \mathrm{y}_{2}\right)$ in ordination space. The Euclidean distance was then regressed on the betadiversity index to estimate $r^{2}$. In order to study the effect of the stressors on the overall phylumlevel community composition, likelihood-ratio-Chi-squared test was used to examine the significant difference between the relative abundances of phyla in the no-stressor enrichment control versus the various enrichments.

Our strategy to achieve the second goal (i.e. understanding the contribution of the rare biosphere to environmental stressors) is based on the identification of the abundant members of the community post stressor application in different enrichments, and tracing their origins to the various fractions of the community in the no-stressor control incubation. This allows for quantifying the contribution of the rare biosphere in the no-stressor control incubation to the 
174 microbial community that developed post application of stressors. The promotion of specific

175 members of the rare biosphere in the control incubation to become abundant members in the

176

177

178

179 post-stressor enrichments communities suggests a role for such rare biosphere members in responding to environmental stressors. Also, the relative abundances of such promoted members in the post-stressor enrichment community could be regarded as an indicator of the magnitude of importance of this promotion process.

Details of the analysis conducted are shown in Figure 1. First, each sequence affiliated with the no-stressor control incubation was classified into either "abundant", "unique rare", or "non-unique rare" classes using the classification criteria detailed before (Youssef et al. 2010). Briefly, "abundant" refers to all sequences binned into OTUs with $>10$ representatives, "unique rare" refers to all sequences binned into OTUs with $\leq 10$ representatives and that are phylogenetically distinct from more abundant members of the community $(>85 \%$ sequence similarity), while "non-unique rare" refers to all sequences binned into OTUs with $\leq 10$ representatives and that are phylogenetically similar to more abundant members of the community (Youssef et al. 2010). We then queried all abundant ( $\mathrm{n}>10) \mathrm{OTU}_{0.03}$ representatives from individual post-stressor treatments against all sequences recovered from the no-stressor control experiment (14,071 sequences) using local Blastn. Identification of the best "hit" for each abundant $\mathrm{OTU}_{0.03}$ representative in the post-stressor incubations allowed us to trace its origin in the no-stressor control incubation into either "originating from abundant", "originating from unique rare", or "originating from non-unique rare" sequence. Abundant post-stressor OTUs originating from an "abundant" control sequence represent the "no change" effect, where an abundant member remained abundant post-stressor application. Abundant post-stressor OTUs originating from a "rare" control sequence represent the "promotion" effect, where a rare 
197

198

199

member in the no-stressor incubation control was promoted to a more abundant OTU poststressor. This latter group could be further subdivided into two distinct categories: Abundant post-stressor OTUs "originating from non-unique rare" control sequence, and abundant poststressor OTUs "originating from unique rare" control sequence. Based on these results, we calculated the percentage of post-stressor abundant OTUs that were "originating from abundant", "originating from unique rare", or "originating from non-unique rare" sequences, and correlated these values to the severity of enrichment (salinity or temperature) using Pearson correlations. Controlling the false rate of discovery using Benjamini-Hochberg adjustment. P-values were calculated for all Pearson correlation coefficients. Due to the large number of correlations conducted in this study, and to avoid any spurious correlations that might arise by chance (rejecting the null hypothesis due to a high p-value, when in fact the null hypothesis is true), we used the Benjamini-Hochberg procedure (Benjamini \& Hochberg 1995) to adjust for the false discovery rate. The command p.adjust in $\mathrm{R}$ was used. 
211 Overall sequencing results. A total of 74,017 high quality sequences were obtained in this 212 study. Estimates of diversity, species richness, evenness, and beta diversity at the species level $213\left(\mathrm{OTU}_{0.03}\right)$ in various enrichments are shown in Table 1. As expected, multiple sequences

214

affiliated with various sulfur-metabolizing lineages were identified within the datasets, including members of the sulfate- and sulfur-reducing $\delta$-Proteobacteria, chemolithotrophic sulfur-oxidizing $\beta$ - and $\gamma$-Proteobacteria, as well as purple ( $\alpha$ - and $\gamma$-Proteobacteria) and green (Chloroflexi and Chlorobia) sulfide- and sulfur-oxidizing anoxygenic phototrophs (Table S1).

Effect of environmental stressors on overall microbial community. In general, the increase in temperature negatively affected the community diversity (measured as rarefaction curve rank, Figure S1) (Pearson correlation coefficient $=-0.67, \mathrm{P}=0.17$, Figure S2a), with the magnitude of diversity loss directly correlated to the prevailing temperature. On the other hand, all salinity treatments resulted in an increase in community diversity compared to the no-treatment control, as evident by diversity ranking estimates obtained (Table 1). However, that increase was not directly correlated to increments in salinity (Pearson correlation coefficient $=0.06$, Figure S2b).

At the phylum level, 50 distinct bacterial phyla and candidate phyla were identified in this study. The highest phylum level diversity was observed in sediments obtained directly from the spring prior to enrichment (50 phyla), followed by the no-treatment control enrichment (35 phyla, Table S1, Figure 2). Similar to diversity statistics at the putative species level, the number of phyla identified was negatively correlated to the enrichment temperature (Pearson correlation coefficient $=-0.66, \mathrm{P}=0.17$, Figure $\mathrm{S} 1$ ), while salinity had no clear effect on phylum level diversity (Pearson correlation coefficient $=-0.09)($ Table 1$)$. 
the relative abundances of phyla in the no-treatment enrichment control versus the various

enrichments. This method failed to identify any significant difference (likelihood ratio $\chi^{2}=99.4$, $p=1)$. Nevertheless, Pearson correlations between specific phyla relative abundance in the various enrichments, and the enrichment condition (salinity or temperature) identified the following patterns (Figure 2 and Table S1). The increase in the enrichment temperature was positively correlated to the percentage abundance of Firmicutes, Aminicenantes (candidate division OP8), Parcubacteria (candidate division OD1), and Thermotogae (Pearson correlation coefficients $=0.98(\mathbf{P}=\mathbf{0 . 0 0 0 5}), 0.9(\mathbf{P}=\mathbf{0 . 0 1 4}), 0.98(\mathbf{P}=\mathbf{0 . 0 0 0 3})$, and $0.75(\mathbf{P}=\mathbf{0 . 1})$, respectively, Figure S3), and negatively correlated to the percentage abundances of Bacteroidetes, Marinimicrobia (candidate division SAR406), Verrucomicrobia and Latescibacteria (candidate division WS3) (Pearson correlation coefficients $=-0.9(\mathbf{P}=\mathbf{0 . 0 1 6}),-0.75(\mathbf{P}=\mathbf{0 . 1}),-0.82(\mathbf{P}=\mathbf{0 . 0 6})$, and $-0.75(\mathbf{P}=\mathbf{0 . 1})$, respectively, Figure S3). The increase in enrichment salt concentration was positively correlated to the percentage abundance of Firmicutes, and Thermotogae (Pearson correlation coefficients $=0.53(\mathrm{P}=0.258)$, and $0.52(\mathrm{P}=0.269)$, respectively, Figure $\mathrm{S} 3)$, and negatively correlated to the percentage abundances of Verrucomicrobia and Latescibacteria (candidate division WS3) $($ Pearson correlation coefficients $=-0.6(\mathbf{P}=\mathbf{0 . 1 8})$, and $-0.73(\mathbf{P}=\mathbf{0 . 0 8})$, respectively, Figure S3). All P-values shown in boldface were significant following BenjaminiHochberg adjustment (Benjamini \& Hochberg 1995).

Shifts in dominant microbial populations post stressors application. To examine the impact of stressors on the microbial community, we examined the occurrence and magnitude of shifts in the structure of abundant community members post enrichments, compared to the no-treatment control. Multiple lines of evidence suggest a shift in the abundant community post stressors 
application: The proportion of sequences belonging to abundant OTUs ( $\mathrm{n}>10)$ decreased post enrichment (Table 1). In addition, high levels of beta diversity were observed between the notreatment incubation and all salinity and temperature enrichments. The abundant no-treatment community showed Morisita Horn indices of $0.63 \pm 0.07$, and $0.57 \pm 0.3$ for all possible pair wise comparisons with the salinity, and temperature post-stressors abundant communities, respectively. Indeed, non-metric multidimensional scaling plot using Morisita-Horn indices clearly shows a shift in the abundant community structure following stress application (Figure 3). Finally, phylogenetic affiliations of abundant members of the community following application of stressors showed marked differences from those in the no treatment control (Figure 4).

\section{Origins of the abundant community members following enrichment support a role for the}

rare biosphere in responding to environmental stressors. We hypothesized that the differences observed in dominant members of the community following application of stressors are due to recruitment of organisms from the rare biosphere to become part of the abundant members of the communities. To this end, we sought to identify the origin of all members of the abundant community post stressors application (Figure 1) and determine their origin (abundant, rare unique, and rare non unique) in the no-treatment control community. Our analysis identified three distinct patterns (Table 2): First, post-enrichment abundant OTUs that were similar to "abundant" sequences in the no-treatment control. This group constituted $36.7-91.1 \%$ of the community in various enrichments. As expected from the community structure analysis, the highest percentages were encountered with the $28^{\circ} \mathrm{C}$ and $30^{\circ} \mathrm{C}$ incubations, since the abundant community from these temperature incubations was very similar to the no-treatment control (Figure 3). The percentage of this group decreased as the enrichment salinity, and temperature increased (Pearson correlation coefficient $=-0.6$, and $-0.86(\mathrm{P}=0.07)$ for salinity, and 
temperature, respectively, Figure S4). The second group constitutes abundant OTUs in the enrichments (8.9-63.3\%) that were recruited from the no-treatment control rare biosphere, providing direct evidence that the rare biosphere could act as a backup system to respond to environmental stressors. Within this group, we differentiate between two distinct fractions: A. Post-enrichment abundant OTUs that were promoted from "rare non-unique" sequences in the no-treatment control, i.e. rare members of the original community phylogenetically similar to more abundant members of the community. Of the total number of abundant OTUs, this fraction constituted $2.6-10.2 \%$ in various conditions. This percentage decreased as the enrichment salinity, and temperature increased (Pearson correlation coefficient $=-0.86$ for both salinity $(\mathrm{P}=0.035)$, and temperature $(\mathrm{P}=0.076)$, Figure $\mathrm{S} 4)$. And B. Post-enrichment abundant OTUs that were promoted from "rare unique" sequences in the no-treatment control, i.e. rare members of the original community phylogenetically distinct from more abundant members of the community. Of the total number of abundant OTUs, this fraction constituted $1.8-60.7 \%$ of abundant OTUs in various conditions. This percentage increased as the enrichment salinity, and temperature increased (Pearson correlation coefficient $=0.85(\mathrm{P}=0.039)$, and $0.86(\mathrm{P}=0.072)$ for salinity, and temperature, respectively Figure S4). Therefore, we argue that, while both factions of the rare biosphere are important in responding to changes in environmental conditions and both act to recruit members to the abundant community, the magnitude of contribution of the "non-unique" rare biosphere to the promotion process was less significant. On the other hand, the "unique" rare biosphere seemed to contribute to the promotion process both when the magnitude of environmental stressors applied was slight, e.g. similar to what would happen during diurnal variation in salinity and temperature, as well as severe, e.g. similar to what would be encountered during seasonal variation in temperature or salinity, or following a drastic change in 
environmental condition, e.g. drought or fire. This latter effect is so evident in the $10 \%$ salt, and

\section{2}

the $70^{\circ} \mathrm{C}$ incubation, where $55 \%$, and $61 \%$, respectively of the abundant community was

303 recruited from the "unique" rare biosphere (Table 2).

304

305 


\section{Discussion}

In this study, we experimentally evaluated the response of microbial community from Zodletone spring source sediments to environmental stressors (various levels of elevated salinities or temperatures), with emphasis on understanding the role of the rare biosphere in the process. We demonstrate that rare bacterial taxa could be promoted to abundant members of the community following environmental manipulations. The magnitude of this promotion process is directly proportional to the severity of the stress applied. Finally, rare members that are phylogenetically distinct from abundant taxa in the original sample (unique rare biosphere) play a more important role in microbial community response to environmental stressors. The results are in agreement with several previously proposed ideas regarding the function and maintenance of the rare biosphere. Prior studies have speculated that the rare biosphere acts as a backup system that preserves microbial community-level function in face of stress (Elshahed et al. 2008; Lynch \& Neufeld 2015; Reid \& Buckley 2011). This fraction of the rare biosphere has been referred to as "conditionally rare taxa". The occurrence of conditionally rare taxa in multiple environments has recently been documented (Aanderud et al. 2015; Shade \& Gilbert 2015; Shade et al. 2014; Sjostedt et al. 2012; Taylor et al. 2013; Walke et al. 2014). These taxa show cyclical low abundance until conditions become favorable, where they respond to the change and increase in abundance. Previous studies showed that such taxa could make up to $28 \%$ of the community (Shade et al. 2014). Conditionally rare taxa contribute greatly to the diversity of an ecosystem. The presence of a diverse low-abundance fraction increases the ecosystem's ability to maintain its functions during environmental changes (Shade et al. 2012; Yachi \& Loreau 1999). In the current study, rare taxa in the no-treatment control incubation that were promoted to become abundant members in different incubations constituted $8.9-63.3 \%$ of 
the microbial community under various conditions of elevated temperatures or salinity. This provides direct evidence that this fraction in the rare biosphere acts as a backup system.

Within these conditionally rare taxa that increased in abundance in response to environmental stress, we differentiate between two different fractions, those that were promoted from "rare unique" sequences in the no-treatment control, i.e. rare members of the original community phylogenetically distinct from more abundant members of the community, and those that were promoted from "rare non-unique" sequences in the no-treatment control i.e. rare members of the original community phylogenetically similar to more abundant members within the original community. Our results suggest that the rare unique members of the microbial community actively contributes to the ecosystem's response to stress, since the majority of conditionally rare taxa identified in various enrichments were unique in the no-treatment control incubation, e.g. $37 \%$ to $\sim 61 \%$ for temperature incubations $\geq 32^{\circ} \mathrm{C}$, and $37 \%$ to $\sim 55 \%$ for salt incubations (Table 2). The magnitude of contribution of the rare unique fraction to the abundant community increased with the severity of stress (i.e. with the increase in salinity as well as temperature of enrichment). Interestingly, the abundant community in the most severe "unnatural" condition (the enrichment at $70^{\circ} \mathrm{C}$ ) was mostly $(60.7 \%)$ made-up from representatives of the "unique" rare biosphere (Table 2), confirming what was shown before in arctic marine sediments (Hubert et al. 2009), and freshwater stratified lakes (Shade et al. 2012) that a fraction of the conditionally rare taxa exhibit greatly reduced metabolic activity under the natural environmental conditions but is able to exploit the "forced" manipulation of conditions (e.g. high temperature incubations of arctic sediments, or complete anoxic conditions in the hypolimnion layers of the lake) and become abundant. The "non-unique" rare biosphere, on the other hand, did not significantly contribute to the abundant community post-stressors, with the 
352 highest representation of its members to the post-stress abundant community being $10 \%$ in the $3531 \%$ salt enrichment (Table 2), and the magnitude of its contribution to the abundant community 354 decreasing with the severity of stress.

355

356

In addition to conditionally rare taxa, the post-stress abundant community was also partly made-up (36.7-91.1\%) of previously abundant members in the no-treatment control, where microorganisms capable of coping with the new condition (increased salt or temperature) remained abundant. It is telling that the highest magnitude of contribution of the pre-stress abundant members to the post-stress abundant community was observed in the $28^{\circ} \mathrm{C}(91.1 \%)$ and $30^{\circ} \mathrm{C}(85.6 \%)$ enrichments, since these two conditions are the closest to the no-treatment control. While those numbers could possibly be inflated by lingering DNA from cells that were originally abundant in the no-treatment control but that lysed or became inactive during enrichment (what has previously been referred to as taphonomic gradient), the observation that the magnitude of contribution of the pre-stress abundant members to the post-stress abundant community decreased with the increase in the severity of stress is expected. This is due to the fact that abundant members of the community under certain conditions are those microorganisms that are most adapted to their current environment, and as the conditions change, those members are also expected to change in abundance and/or metabolic activity. That also explains the difference observed in the abundant community structure pre and post application of stress (Figure 4).

\section{Conclusions}

In conclusion, the current study provided direct evidence for the contribution of members of the rare biosphere to the abundant community post environmental stress, and hence confirming the notion that a fraction of the rare biosphere acts as a backup system that readily 
375 responds to environmental stressors. While we show here, similar to previous studies ((Lynch \&

376 Neufeld 2015), and references within), that the rare biosphere responds to both periodical (e.g.

377 temperature and seasonal changes), as well as drastic (e.g. what would be encountered in drought

378 or fire) changes in the ecosystem conditions, the disproportionate contribution of rare members

379 whose phylogenetic affiliations are distinct when compared to more abundant members of the

380 community has not been shown before and reinforces the phylogenetic and metabolic complexity

381 and diversity of the rare biosphere.

382

383

384

385

386

387

388

389

390

References

391

392

393
Aanderud Z, Jones S, Fierer N, and Lennon JT. 2015. Resuscitation of the rare biosphere contributes to pulses of ecosystem activity. Front Microbiol 6:24.

Alonso-Saez L, Diaz-Perez L, and Moran XA. 2015. The hidden seasonality of the rare biosphere in coastal marine bacterioplankton. Environ Microbiol. doi: 10.1111/14622920.12801 . 
Alonso-Saez L, Zeder M, Harding T, Pernthaler J, Lovejoy C, Bertilsson S, and Pedros-Alio C. 2014. Winter bloom of a rare betaproteobacterium in the Arctic Ocean. Front Microbiol $5: 425$.

Anderson MJ, and Millar RB. 2004. Spatial variation and effects of habitat on temperate reef fish assemblages in northeastern New Zealand. J Exp Marine Biol Ecol 305:191-221.

Anderson RE, Sogin ML, and Baross JA. 2015. Biogeography and ecology of the rare and abundant microbial lineages in deep-sea hydrothermal vents. FEMS Microbiol Ecol 91:111.

Ashby MN, Rine J, Mongodin EF, Nelson KE, and Dimster-Denk D. 2007. Serial analysis of rRNA genes and the unexpected dominance of rare members of microbial communities. Appl Environ Microbiol 73:4532-4542.

Benjamini Y, and Hochberg Y. 1995. Controlling the false discovery rate: a practical and powerful approach to multiple testing. J Roy Stat Soc B 57: 289-300.

Besemer K, Peter H, Logue JB, Langenheder S, Lindstrom ES, Tranvik LJ, and Battin TJ. 2012. Unraveling assembly of stream biofilm communities. ISME J 6:1459-1468.

Bowen JL, Morrison HG, Hobbie JE, and Sogin ML. 2012. Salt marsh sediment diversity: a test of the variability of the rare biosphere among environmental replicates. ISME J 6:20142023.

Bühring SI, Sievert SM, Jonkers HM, Ertefai T, Elshahed MS, Krumholz LR, and Hinrichs KU. 2011. Insights into chemotaxonomic composition and carbon cycling of phototrophic communities in an artesian sulfur-rich spring (Zodletone, Oklahoma, USA), a possible analog for ancient microbial mat systems. Geobiology 9:166-179. 
Campbell BJ, Yu L, Heidelberg JF, and Kirchman DL. 2011. Activity of abundant and rare bacteria in a coastal ocean. Proc Natl Acad Sci 108:12776-12781.

Caporaso JG, Bittinger K, Bushman FD, DeSantis TZ, Andersen GL, and Knight R. 2010. PyNAST: a flexible tool for aligning sequences to a template alignment. Bioinformatics 26:266-267.

Crump BC, Amaral-Zettler LA, and Kling GW. 2012. Microbial diversity in arctic freshwaters is structured by inoculation of microbes from soils. ISME $J$ 6:1629-1639.

Elshahed MS, Youssef NH, Spain AM, Sheik C, Najar FZ, Sukharnikov LO, Roe BA, Davis JP, Schloss PD, Bailey VL, and Krumholz LR. 2008. Novelty and uniqueness patterns of rare members of the soil biosphere. Appl Environ Microbiol 74:5422-5428.

Galand PE, Casamayor EO, Kirchman DL, and Lovejoy C. 2009. Ecology of the rare microbial biosphere of the Arctic Ocean. Proc Natl Acad Sci USA 106:22427-22432.

Gobet A, Boer SI, Huse SM, van Beusekom JE, Quince C, Sogin ML, Boetius A, and Ramette A. 2012. Diversity and dynamics of rare and of resident bacterial populations in coastal sands. ISME J 6:542-553.

Hubert C, Loy A, Nickel M, Arnosti C, Baranyi C, Brüchert V, Ferdelman T, Finster K, Christensen FM, Rosa de Rezende J, Vandieken V, and Jørgensen BB. 2009. A constant flux of diverse thermophilic bacteria into the cold arctic seabed. Science 325:1541-1544.

Hugoni M, Taib N, Debroas D, Domaizon I, Jouan Dufournel I, Bronner G, Salter I, Agogue H, Mary I, and Galand PE. 2013. Structure of the rare archaeal biosphere and seasonal dynamics of active ecotypes in surface coastal waters. Proc Natl Acad Sci USA 110:60046009. 
440 Jones SE, and Lennon JT. 2010. Dormancy contributes to the maintenance of microbial 441 diversity. Proc Natl Acad Sci USA 107:5881-5886.

442 Lennon JT, and Jones SE. 2011. Microbial seed banks: the ecological and evolutionary 443 implications of dormancy. Nat Rev Microbiol 9:119-130.

444 Liu L, Yang J, Yu Z, and Wilkinson DM. 2015. The biogeography of abundant and rare

Logares R, Audic S, Bass D, Bittner L, Boutte C, Christen R, Claverie JM, Decelle J, Dolan JR, Dunthorn M, Edvardsen B, Gobet A, Kooistra WH, Mahe F, Not F, Ogata H, Pawlowski J, Pernice MC, Romac S, Shalchian-Tabrizi K, Simon N, Stoeck T, Santini S, Siano R, Wincker P, Zingone A, Richards TA, de Vargas C, and Massana R. 2014. Patterns of rare and abundant marine microbial eukaryotes. Curr Biol 24:813-821.

Lynch MD, Bartram AK, and Neufeld JD. 2012. Targeted recovery of novel phylogenetic diversity from next-generation sequence data. ISME J 6:2067-2077.

Lynch MDJ, and Neufeld JD. 2015. Ecology and exploration of the rare biosphere. Nat Rev Micro 13:217-229.

Marchant R, Banat IM, Rahman TJ, and Berzano M. 2002. The frequency and characteristics of highly thermophilic bacteria in cool soil environments. Environ Microbiol 4:595-602.

Pedros-Alio C. 2006. Marine microbial diversity: can it be determined? Trends Microbiol $14: 257-263$.

Pester M, Bittner N, Deevong P, Wagner M, and Loy A. 2010. A 'rare biosphere' microorganism contributes to sulfate reduction in a peatland. ISME J 4:1591-1602. 
Pester M, Knorr KH, Friedrich MW, Wagner M, and Loy A. 2012. Sulfate-reducing microorganisms in wetlands - fameless actors in carbon cycling and climate change. Front Microbiol 3:72.

Reid A, and Buckley M. 2011. The rare biosphere. American Academy of Microbiology.

Reveillaud J, Maignien L, Murat Eren A, Huber JA, Apprill A, Sogin ML, and Vanreusel A. 2014. Host-specificity among abundant and rare taxa in the sponge microbiome. ISME $J$ 8:1198-1209.

Schloss PD, Westcott SL, Ryabin T, Hall JR, Hartmann M, Hollister EB, Lesniewski RA, Oakley BB, Parks DH, Robinson CJ, Sahl JW, Stres B, Thallinger GG, Van Horn DJ, and Weber CF. 2009. Introducing mothur: open-source, platform-independent, communitysupported software for describing and comparing microbial communities. Appl Environ Microbiol 75:7537-7541.

Senko JM, Campbell BS, Henriksen JR, Elshahed MS, Dewers TA, and Krumholz LR. 2004. Barite deposition resulting from phototrophic sulfide-oxidizing bacterial activity. Geochim Cosmochim Acta 68:773-780.

Shade A, and Gilbert JA. 2015. Temporal patterns of rarity provide a more complete view of microbial diversity. Trends Microbiol doi: 10.1016/j.tim.2015.01.007.

Shade A, Jones SE, Caporaso JG, Handelsman J, Knight R, Fierer N, and Gilbert JA. 2014. Conditionally rare taxa disproportionately contribute to temporal changes in microbial diversity. MBio 5:e01371-01314.

Shade A, Peter H, Allison SD, Baho D, Berga M, Buergmann H, Huber DH, Langenheder S, Lennon JT, Martiny JB, Matulich KL, Schmidt TM, and Handelsman J. 2012. Fundamentals of microbial community resistance and resilience. Front Microbiol 3:417. 
Sjostedt J, Koch-Schmidt P, Pontarp M, Canback B, Tunlid A, Lundberg P, Hagstrom A, and Riemann L. 2012. Recruitment of members from the rare biosphere of marine bacterioplankton communities after an environmental disturbance. Appl Environ Microbiol 78:1361-1369.

Sogin ML, Morrison HG, Huber JA, Welch DM, Huse SM, Neal PR, Arrieta JM, and Herndl GJ. 2006. Microbial diversity in the deep sea and the underexplored "rare biosphere". Proc Natl Acad Sci 103:12115-12120.

Taylor MW, Tsai P, Simister RL, Deines P, Botte E, Ericson G, Schmitt S, and Webster NS. 2013. 'Sponge-specific' bacteria are widespread (but rare) in diverse marine environments. ISME J 7:438-443.

Walke JB, Becker MH, Loftus SC, House LL, Cormier G, Jensen RV, and Belden LK. 2014. Amphibian skin may select for rare environmental microbes. ISME J 8:2207-2217.

Wilhelm L, Besemer K, Fasching C, Urich T, Singer GA, Quince C, and Battin TJ. 2014. Rare but active taxa contribute to community dynamics of benthic biofilms in glacier-fed streams. Environ Microbiol 16:2514-2524.

Yachi S, and Loreau M. 1999. Biodiversity and ecosystem productivity in a fluctuating environment: The insurance hypothesis. Proc Natl Acad Sci 96:1463-1468.

Youssef N, Steidley BL, and Elshahed MS. 2012. Novel high-rank phylogenetic lineages within a sulfur spring (Zodletone Spring, Oklahoma), revealed using a combined pyrosequencing-sanger approach. Appl Environ Microbiol 78:2677-2688.

Youssef NH, Couger MB, and Elshahed MS. 2010. Fine-scale bacterial beta diversity within a complex ecosystem (Zodletone Spring, OK, USA): the role of the rare biosphere. PLoS One 5:e12414. 
508 Youssef NH, and Elshahed MS. 2009. Diversity rankings among bacterial lineages in soil. ISME 509 $J 3: 305-313$. 
$\underline{\text { Figure legends }}$

511 Figure 1. A flowchart depicting the experimental design. Abundant post-disturbance OTUs 512 originating from an "abundant" control sequence represent the "no change" effect, where an 513 abundant member remained abundant post-disturbance. Abundant post-disturbance OTUs 514 originating from a "rare" control sequence represent the "promotion" effect, where a rare

member of the no-stressor control was promoted to a more abundant OTU post-disturbance. This

latter group could be further subdivided into two distinct categories: a. Abundant post-

disturbance OTUs “originating from non-unique rare" control sequence. And b. Abundant post-

disturbance OTUs "originating from unique rare" control sequence. * salinity percentage

indicates salt concentration above ambient values (calculated at $0.9 \%$ ).

Figure 2. Heatmap of the percentage abundance of phyla encountered in the different enrichments versus the un-incubated and the no-stressor control. Gracilibacteria denotes candidate division GN02, Marinimicrobia denotes candidate division SAR406, Aminicenantes denotes candidate division OP8, Atribacteria denotes candidate division OP9, Parcubacteria denotes candidate division OD1, Saccharibacteria denotes candidate division TM7, and Latescibacteria denotes candidate division WS3. Other CD denotes other candidate divisions including SPAM, AD3, CV51, KSB3, NC10, WS6, WPS-2, BRC1, SR1, WS6, ZB3, H-178, GN04, Hyd24-12, OP1, and OP11.

Figure 3. Non-metric multidimensional scaling based on pairwise Morisita-Horn dissimilarity indices between abundant members of all enrichments (stress value $=0.109$, the two axes explain $79.5 \%$ of the variance). Each symbol represents one enrichment condition. The temperature enrichments abundant communities are shown by (↔) and each is labeled with the temperature. The salinity enrichments abundant communities are shown by ( The no-treatment control 
abundant community is shown by $(\oplus)$. All salinity post-disturbance abundant communities clustered together away from the no-treatment control incubation, while the effect of temperature incubations on the abundant community structure was more complex. Abundant communities following incubations at $28^{\circ} \mathrm{C}$ and $30^{\circ} \mathrm{C}$ had a structure more similar to the no-treatment control (which was incubated at room temperature) than the abundant community following incubation at $32^{\circ} \mathrm{C}$. The most drastic effect on the abundant community structure was the $70^{\circ} \mathrm{C}$ incubation. The $70^{\circ} \mathrm{C}$ abundant community clustered alone to the far left of the NMDS plot reflecting a major shift in community structure, and showed an average Morisita Horn index of $0.94 \pm 0.04$. Figure 4. Effect of temperature (A), and salinity (B) on the phylogeny of abundant OTUs $\mathrm{s}_{0.03}$ following enrichment. The Y-axis (logarithmic scale) shows the percentage of abundant OTUs affiliated with each phylum on the X-axis as a fraction of all abundant OTUs encountered in the post-disturbance enrichment. At the species level $\left(\mathrm{OTU}_{0.03}\right)$, abundant OTUs $(\mathrm{n}>10)$ in the notreatment control belonged to the phyla Acidobacteria, Actinobacteria, Bacteroidetes, Chloroflexi, Firmicutes, Marinimicrobia (candidate division SAR406), Nitrospirae, Aminicenantes (candidate division OP8), Atribacteria (candidate division OP9), Proteobacteria, Spirochaetes, and Thermotogae. This phylogenetic profile of abundant OTUs was maintained in all enrichment conditions with the following exceptions. The increase in the salt concentration of the enrichment (A): 1. Recruited sequences belonging to Chlorobi (1\% salt enrichment), and Gemmatimonadetes ( $2 \%$ salt enrichment) to the abundant biosphere. 2. Demoted all sequences belonging to Marinimicrobia (candidate division SAR406), Nitrospirae, and Atribacteria (candidate division OP9) to the rare biosphere. 3. Resulted in the decrease in the number of abundant OTUs belonging to Bacteroidetes, and Spirochaetes, and the increase in the number of abundant OTUs belonging to Firmicutes, Chloroflexi, Actinobacteria, and Thermotogae. On the 
556 other hand, the increase in the enrichment incubation temperature (B): 1. Recruited sequences

557 belonging to Chlorobi $\left(32^{\circ} \mathrm{C}\right.$ enrichment) to the abundant biosphere. 2. Demoted all sequences

558 belonging to Chloroflexi, and Nitrospirae to the rare biosphere. 3. Resulted in the decrease in the

559 number of abundant OTUs belonging to Bacteroidetes, and the increase in the number of

560 abundant OTUs belonging to Firmicutes, and Actinobacteria. Also, a significant increase in the

561 percentage of abundant OTUs belonging to Aminicenantes (candidate division OP8) was

562 observed in the $70^{\circ} \mathrm{C}$ enrichment, and a significant increase in the percentage of abundant OTUs

563 belonging to Atribacteria (candidate division OP9) was observed in the $28^{\circ} \mathrm{C}$ enrichment. 


\section{Figure 1 (on next page)}

A flowchart depicting the experimental design.

Figure 1. A flowchart depicting the experimental design. Abundant post-disturbance OTUs originating from an "abundant" control sequence represent the "no change" effect, where an abundant member remained abundant post-disturbance. Abundant post-disturbance OTUs originating from a "rare" control sequence represent the "promotion" effect, where a rare member of the no-stressor control was promoted to a more abundant OTU post-disturbance. This latter group could be further subdivided into two distinct categories: a. Abundant postdisturbance OTUs "originating from non-unique rare" control sequence. And b. Abundant post-disturbance OTUs "originating from unique rare" control sequence. * salinity percentage indicates salt concentration above ambient values (calculated at $0.9 \%$ ). 
Create microcosms under anaerobic condition

\begin{tabular}{|c|l|l|}
\hline Salinity* & \multicolumn{2}{|c|}{$l$} \\
$1 \%, 2 \%, 3 \%$, & $\begin{array}{l}\text { Temperature } \\
28^{\circ} \mathrm{C}, 30^{\circ} \mathrm{C}, 32^{\circ} \mathrm{C},\end{array}$ & $\begin{array}{l}\text { Control group } \\
\text { Room temperature } \\
\text { no salt }\end{array}$ \\
\hline $4 \%, 10 \%$ & $70^{\circ} \mathrm{C}$ & \\
\hline
\end{tabular}

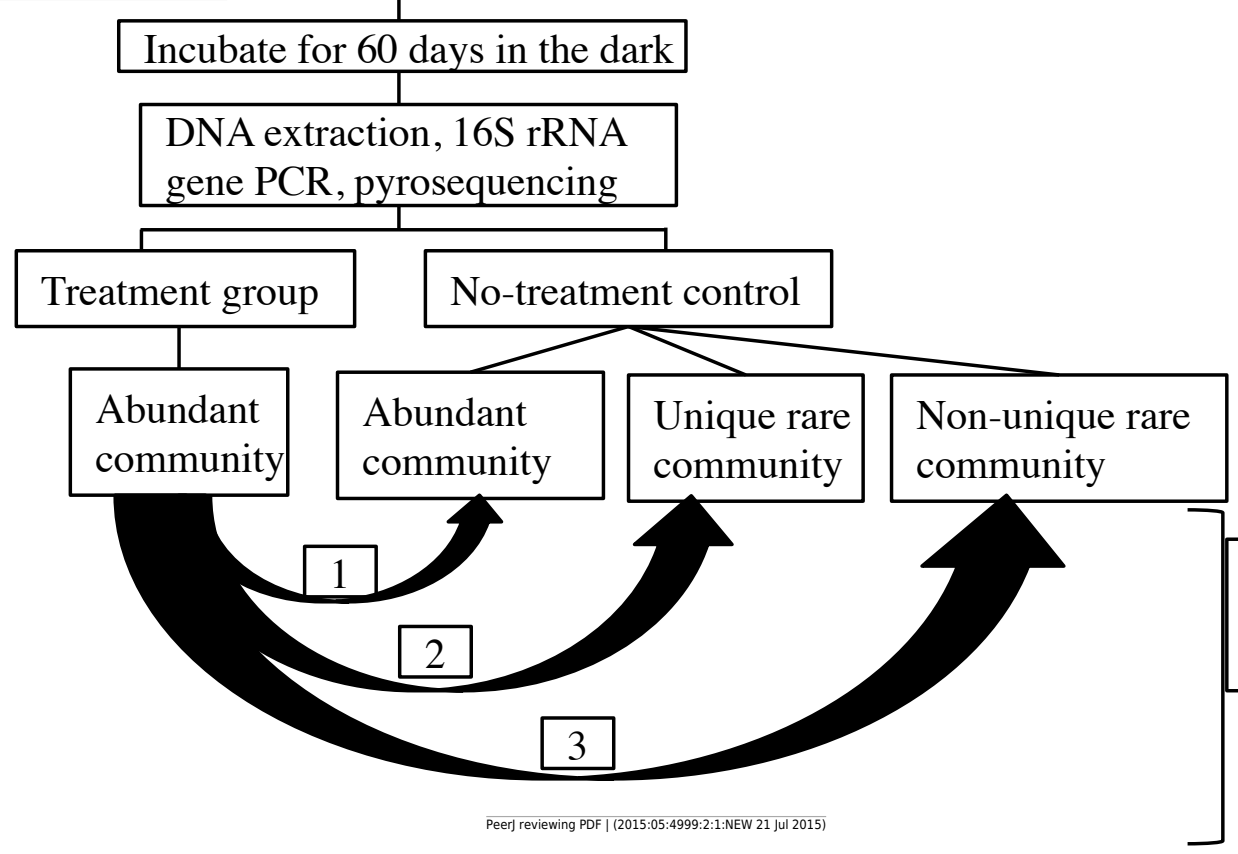

Blastn to find origins of the abundant community in the treatments

\begin{tabular}{|l|}
\hline 1. "No change" effect \\
\hline 2. "Promotion" effect \\
\hline 3. "Promotion" effect \\
\hline
\end{tabular}


Figure 2 (on next page)

Heatmap of the percentage abundance of phyla encountered in the different enrichment.

Figure 2. Heatmap of the percentage abundance of phyla encountered in the different enrichments versus the un-incubated and the no-stressor control. Gracilibacteria denotes candidate division GN02, Marinimicrobia denotes candidate division SAR406, Aminicenantes denotes candidate division OP8, Atribacteria denotes candidate division OP9, Parcubacteria denotes candidate division OD1, Saccharibacteria denotes candidate division TM7, and Latescibacteria denotes candidate division WS3. Other CD denotes other candidate divisions including SPAM, AD3, CV51, KSB3, NC10, WS6, WPS-2, BRC1, SR1, WS6, ZB3, H-178, GN04, Hyd24-12, OP1, and OP11. 

Gamma_proteobacteria Epsilon_proteobacteria Beta_proteobacteria Alpha_proteobacteria Delta_proteobacteria Planctomycetes Atribacteria Aminicenantes Nitrospirae Marinimicrobia Lentisphaerae Gracilibacteria Gemmatimonadetes -

Fusobacteria -

Firmicutes Fibrobacteres Elusimicrobia Cyanobacteria Chloroflexi Chlorobi Caldithrix Bacteroidetes Armatimonadetes Actinobacteria Acidobacteria -

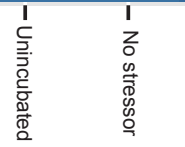

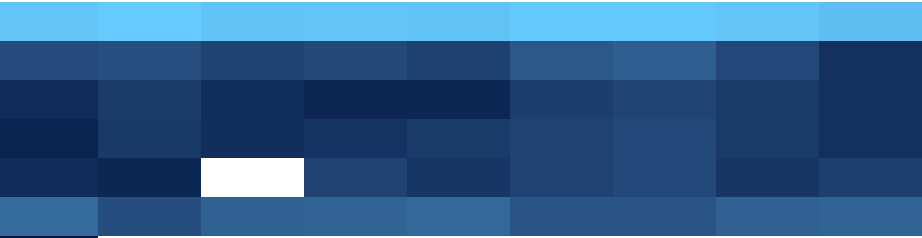

Controls

Abundance 16.0000 1.0000 0.0625 


\section{Figure $\mathbf{3}$ (on next page)}

Non-metric multidimensional scaling based on pairwise Morisita-Horn dissimilarity indices between abundant members of all enrichments.

Figure 3. Non-metric multidimensional scaling based on pairwise Morisita-Horn dissimilarity indices between abundant members of all enrichments (stress value $=0.109$, the two axes explain $79.5 \%$ of the variance). Each symbol represents one enrichment condition. The temperature enrichments abundant communities are shown by $(\square)$ and each is labeled with the temperature. The salinity enrichments abundant communities are shown by $\left({ }^{-}\right)$. The notreatment control abundant community is shown by (i). All salinity post-disturbance abundant communities clustered together away from the no-treatment control incubation, while the effect of temperature incubations on the abundant community structure was more complex. Abundant communities following incubations at $28^{\circ} \mathrm{C}$ and $30^{\circ} \mathrm{C}$ had a structure more similar to the no-treatment control (which was incubated at room temperature) than the abundant community following incubation at $32^{\circ} \mathrm{C}$. The most drastic effect on the abundant community structure was the $70^{\circ} \mathrm{C}$ incubation. The $70^{\circ} \mathrm{C}$ abundant community clustered alone to the far left of the NMDS plot reflecting a major shift in community structure, and showed an average Morisita Horn index of 0.94 \pm 0.04 . 


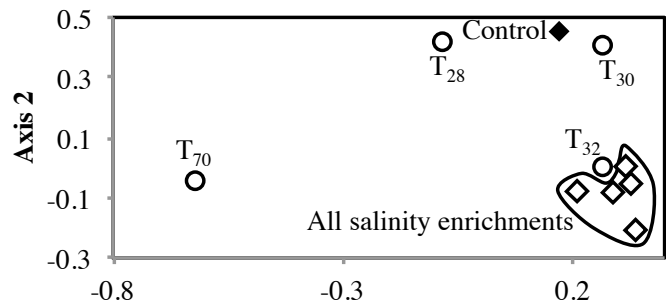

Axis 1 


\section{4}

Effect of salinity, and temperature on the phylogeny of abundant OTUs $\mathrm{S}_{0.03}$ following enrichment.

Figure 4. Effect of temperature (A), and salinity (B) on the phylogeny of abundant OTUS $\mathrm{S}_{0.03}$ following enrichment. The $Y$-axis (logarithmic scale) shows the percentage of abundant OTUs affiliated with each phylum on the X-axis as a fraction of all abundant OTUs encountered in the post-disturbance enrichment. At the species level $\left(\mathrm{OTU}_{0.03}\right)$, abundant OTUs $(n>10)$ in the no-treatment control belonged to the phyla Acidobacteria, Actinobacteria, Bacteroidetes, Chloroflexi, Firmicutes, Marinimicrobia (candidate division SAR406), Nitrospirae, Aminicenantes (candidate division OP8), Atribacteria (candidate division OP9), Proteobacteria, Spirochaetes, and Thermotogae. This phylogenetic profile of abundant OTUs was maintained in all enrichment conditions with the following exceptions. The increase in the salt concentration of the enrichment (A): 1. Recruited sequences belonging to Chlorobi ( $1 \%$ salt enrichment), and Gemmatimonadetes ( $2 \%$ salt enrichment) to the abundant biosphere. 2. Demoted all sequences belonging to Marinimicrobia (candidate division SAR406), Nitrospirae, and Atribacteria (candidate division OP9) to the rare biosphere. 3. Resulted in the decrease in the number of abundant OTUs belonging to Bacteroidetes, and Spirochaetes, and the increase in the number of abundant OTUs belonging to Firmicutes, Chloroflexi, Actinobacteria, and Thermotogae. On the other hand, the increase in the enrichment incubation temperature (B): 1. Recruited sequences belonging to Chlorobi (32으 $\mathrm{C}$ enrichment) to the abundant biosphere. 2. Demoted all sequences belonging to Chloroflexi, and Nitrospirae to the rare biosphere. 3. Resulted in the decrease in the number of abundant OTUs belonging to Bacteroidetes, and the increase in the number of abundant OTUs belonging to Firmicutes, and Actinobacteria. Also, a significant increase in the percentage of abundant OTUs belonging to Aminicenantes (candidate division OP8) was observed in the $70^{\circ} \mathrm{C}$ enrichment, and a significant increase in the percentage of abundant OTUs belonging to 
Atribacteria (candidate division OP9) was observed in the $28^{\circ} \mathrm{C}$ enrichment.

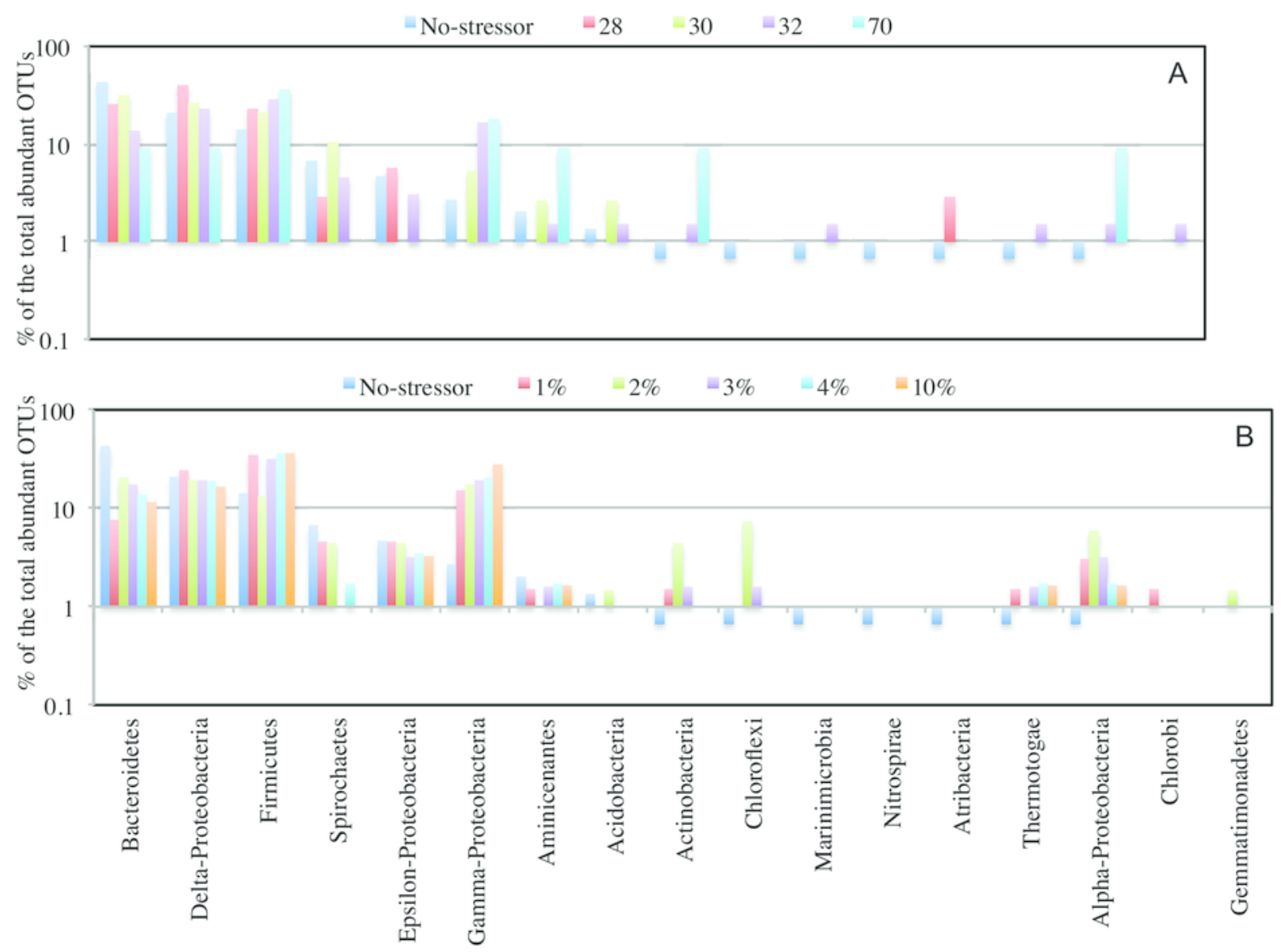




\section{Table $\mathbf{1}$ (on next page)}

Overall diversity estimates in various enrichments versus the no-treatment control incubation.

Table 1. Overall diversity estimates in various enrichments versus the no-treatment control incubation. 
1 Table 1. Overall diversity estimates in various enrichments versus the no-treatment control 2 incubation.

\begin{tabular}{|c|c|c|c|c|c|c|c|c|}
\hline \multirow[t]{2}{*}{ Treatment } & \multirow{2}{*}{$\begin{array}{l}\text { Number } \\
\text { of seqs }\end{array}$} & \multicolumn{6}{|c|}{ Alpha Diversity } & \multirow{2}{*}{\begin{tabular}{|c|c}
\multicolumn{1}{c}{$\begin{array}{c}\text { Beta } \\
\text { diversity }\end{array}$} \\
$\begin{array}{l}\text { Rarefaction } \\
\text { curve rank }\end{array}$ \\
\end{tabular}} \\
\hline & & $\mathrm{OTUs}_{0.03}$ & $\begin{array}{c}\text { Number of } \\
\text { phyla }\end{array}$ & $\begin{array}{c}\% \\
\text { abundant }\end{array}$ & Simpson & Chao & Ace & \\
\hline $\begin{array}{c}\text { No-stressor } \\
\text { control } \\
25^{\circ} \mathrm{C}, 0.9 \% \\
\text { salt }\end{array}$ & 14071 & 4011 & 35 & 50.05 & 0.07 & 11470 & 21911 & 4,1 \\
\hline \multicolumn{9}{|c|}{ Temperature enrichments } \\
\hline $28^{\circ} \mathrm{C}$ & 3783 & 1472 & 29 & 34.68 & 0.14 & 4095 & 7278 & 2 \\
\hline $30^{\circ} \mathrm{C}$ & 3398 & 1393 & 26 & 29.78 & 0.22 & 3924 & 6838 & 3 \\
\hline $32^{\circ} \mathrm{C}$ & 6603 & 2711 & 28 & 37.48 & 0.10 & 9532 & 18095 & 5 \\
\hline $70^{\circ} \mathrm{C}$ & 1788 & 674 & 24 & 38.26 & 0.03 & 1498 & 2430 & 1 \\
\hline Pearson $^{b}$ & & & $-0.66^{*}$ & -0.08 & & & & $-0.67 *$ \\
\hline \multicolumn{9}{|c|}{ Salt enrichments ${ }^{\mathrm{c}}$} \\
\hline $1 \%$ & 6669 & 2732 & 29 & 38.04 & 0.08 & 9643 & 20765 & 4 \\
\hline $2 \%$ & 8317 & 3269 & 34 & 40.21 & 0.07 & 11261 & 25532 & 6 \\
\hline $3 \%$ & 5856 & 2331 & 25 & 39.67 & 0.09 & 8811 & 15943 & 2 \\
\hline $4 \%$ & 6108 & 2563 & 27 & 36.87 & 0.09 & 8727 & 16535 & 5 \\
\hline $10 \%$ & 5511 & 2241 & 32 & 37.69 & 0.11 & 7146 & 14708 & 3 \\
\hline Pearson $^{b}$ & & & -0.09 & -0.54 & & & & 0.06 \\
\hline
\end{tabular}

a: Rarefaction ranks were generated by plotting the rarefaction curves of all enrichments to be compared on the same graph. Enrichments were then ranked from the least diverse (lowermost rarefaction curve, rank 1) to the most diverse (uppermost rarefaction curve, rank 5 for temperature enrichments, or 6 for salinity enrichments). Actual rarefaction curves used for ranking are shown in Figure S1. Two ranks are shown for the no-stressor control incubation corresponding to rarefaction curve ranks compared to the temperature (Figure S1A), and the salinity (Figure S1B) enrichments, respectively.

b: Pearson correlation coefficient between the temperature $\left({ }^{\circ} \mathrm{C}\right) /$ salinity $(\%)$ in the first column and various indices in the table header. P-values for all correlation coefficients are shown in text. *: Significant $\mathrm{p}$-values following Benjamini-Hochberg adjustment for false rate of discovery. c: salinity percentage indicates salt concentration above ambient values (calculated at $0.9 \%$ ). 
Table 2 (on next page)

Tracing the origins of abundant members in elevated salinities and temperature incubations into various fractions within no stressor control incubation.

Table 2. Tracing the origins of abundant members in elevated salinities and temperature incubations into various fractions within no stressor control incubation. 
1 Table 2. Tracing the origins of abundant members in elevated salinities and temperature

2 incubations into various fractions within no stressor control incubation.

3

\begin{tabular}{|l|l|l|l|}
\hline \multirow{2}{*}{} & \multicolumn{3}{|c|}{$\begin{array}{c}\text { \% of abundant OTUs in temperature and } \\
\text { salinity enrichments recruited from }\end{array}$} \\
\cline { 2 - 4 } & Abundant & Unique rare & Non-unique rare \\
\hline Temperature enrichment \\
\hline $28^{\circ} \mathrm{C}$ & 91.14 & 1.77 & 7.08 \\
\hline $30^{\circ} \mathrm{C}$ & 86.96 & 6.01 & 7.03 \\
\hline $32^{\circ} \mathrm{C}$ & 58.62 & 36.93 & 4.44 \\
\hline $70^{\circ} \mathrm{C}$ & 36.7 & 60.67 & 2.63 \\
\hline Pearson $^{\mathrm{b}}$ & $-0.87^{*}$ & $0.86^{*}$ & $-0.86^{*}$ \\
\hline Salt enrichment & \multicolumn{5}{|l|}{} \\
\hline $1 \%$ & 45.44 & 44.34 & 10.21 \\
\hline $2 \%$ & 56.17 & 37.4 & 6.43 \\
\hline $3 \%$ & 47.31 & 46.1 & 6.58 \\
\hline $4 \%$ & 47.78 & 44.76 & 7.46 \\
\hline $10 \%$ & 41.98 & 54.55 & 3.47 \\
\hline Pearson $^{\mathrm{b}}$ & $-0.6^{*}$ & $0.85^{*}$ & $-0.86^{*}$ \\
\hline
\end{tabular}

4 a: The percentage of abundant OTUs in the temperature and salt enrichments that had Blastn best "hits" in the no-treatment control belonging to abundant, unique, and non-unique rare fractions. The percentage of abundant OTUs that were recruited from the unique rare fraction of the notreatment control also includes those abundant OTUs that had no hits in the no-treatment control using the criteria described in Materials and Methods.

b: Pearson correlation coefficient between the temperature $\left({ }^{\circ} \mathrm{C}\right) /$ salinity $(\%)$ in the first column and percentages recruited from the different fractions in the table header. P-values for all correlation coefficients are shown in text. *: Significant p-values following Benjamini-Hochberg adjustment for false rate of discovery.

c: salinity percentage indicates salt concentration above ambient values (calculated at $0.9 \%$ ). 